\title{
Effects of vitamin E supplementation on some pregnancy health indices: a randomized clinical trial
}

This article was published in the following Dove Press journal:

International Journal of General Medicine

14 June 201 I

Number of times this article has been viewed

\author{
Parvin Bastani \\ Kobra Hamdi \\ Fatemeh Abasalizadeh \\ Nazli Navali \\ Women's Reproductive Health \\ Research Center, Tabriz University \\ of Medical Sciences, Tabriz, Iran
}

Correspondence: Parvin Bastani Obstetrics and Gynecology, Tabriz University of Medical Sciences, Tabriz, Iran $\mathrm{Tel}+98(0) 4 I I 556$ I653 Mob +98 914 3II 0142 Email parvinbastani@yahoo.com
Objective: Available evidence about the role of supplementary vitamin E in normal pregnancy is inadequate. This study assessed the potential benefit of vitamin E supplementation on some pregnancy health indices.

Methods: A 1:2 weighted simple randomization technique was used to allocate 104 eligible pregnant women to receive vitamin $\mathrm{E}$ and 168 women (control) not to receive the drug. Treated women received capsules containing 400 IU vitamin E from week 14 of gestation to the end of the pregnancy.

Results: Background variables including maternal age were found to be similarly distributed between the study groups. The rate of maternal and perinatal outcomes including Apgar score and birth weight did not differ significantly between groups. Preeclampsia occurred in $1 \%$ of treated women vs $1.78 \%$ of control women.

Conclusion: Giving supplemental vitamin E from the second trimester of pregnancy did not appear to affect the risk of pregnancy outcomes and occurrence of preeclampsia.

Keywords: antioxidants, vitamin E, pregnancy, supplementation, preeclampsia

\section{Introduction}

Improving maternity outcomes has always been the focus of interest in reproductive health research and researchers have conducted many observational and interventional studies to assess factors or agents affecting pregnancy outcome. Among these are some neonatal outcomes such as Apgar score and birth weight. Preeclampsia has also been the focus of interest in such research. Preeclampsia is a common complication in human pregnancy and is a major source of perinatal morbidity and mortality worldwide. It has been shown recently that preeclampsia may even affect maternal health after pregnancy. ${ }^{1}$

The vital role of vitamin $\mathrm{E}$ in reproduction was first investigated 80 years ago. ${ }^{2}$ Unlike many in vitro studies, promising evidence for the antioxidant properties of vitamin E related to specific outcomes is scarce. ${ }^{3}$ The magic of antioxidants has provided a good motivation for researchers to study effects of antioxidants on pregnancy outcomes and also on preeclampsia. ${ }^{4}$ Vitamin $\mathrm{E}$ has also gained some attention in this regard. Some studies have compared the serum concentrations of vitamin $\mathrm{E}$ in different conditions during pregnancy and some have investigated the role of supplementary vitamin $\mathrm{E}$ in improving pregnancy outcomes. However, a recent systematic review suggested that the available evidence is inadequate to conclude that vitamin E supplementation, either alone or in combination with other supplements, can be beneficial during pregnancy. ${ }^{5}$ 
The aim of this study was to assess the potential benefit of prophylactic antioxidant supplementation (vitamin E) on some pregnancy health indices.

\section{Materials and methods}

A randomized clinical trial was conducted from January 2007 to February 2008 at the Women's Hospital of Tabriz University of Medical Sciences, Iran. Eligible women had singleton gestation with normal blood pressure both at the first measurement in pregnancy and repeated again at trial enrollment.

Eligible women with a gestational age $<14$ weeks, who attended the maternity outpatient clinic of the Women's Hospital for routine check-up, were randomly assigned to two groups. A 1:2 weighted simple randomization technique was used to allocate the patients to receive either vitamin $\mathrm{E}$ or not to receive the drug (control group). To assign patients to each group, an assignment list was produced using the random number generator rand function in Microsoft ${ }^{\circledR}$ Excel. Women in the vitamin E group received capsules containing 400 IU vitamin E (RRR-alpha-tocopherol acetate; Zahravi Pharmaceutical Company, Tabriz, Iran) from week 14 of gestation to delivery time. They were asked to swallow the capsules without crushing or chewing them.

Women with any of the following were ineligible: known multiple pregnancies; specific contraindications to vitamin $\mathrm{E}$ therapy such as hemochromatosis or anticoagulant therapy; ${ }^{4}$ a body mass index (BMI) $<20$ or $>29$; history of hypertension and proteinuria before conception or before 20 weeks of gestation.

Gestational age was calculated based on the last menstrual period date and confirmed by ultrasonography in early pregnancy. The participant was seated for 5 minutes, and then the average of two consecutive measurements taken 3 minutes apart was recorded. Preeclampsia was diagnosed according to the following strict criteria: a systolic blood pressure of $\geq 140 \mathrm{mmHg}$, or a diastolic blood pressure of $\geq 90 \mathrm{mmHg}$, on two occasions at least 6 hours apart occurring after the 20th week of pregnancy in women with previously normal blood pressure, plus detectable urinary protein ( $\geq 1+$ by dipstick or $\geq 0.3$ g per 24 hours).

SPSS statistical software package (v 16.0; SPSS Inc, Chicago, IL) was used to analyze data. Treatment group comparisons were summarized as differences in means or risk ratios and independent sample $t$-tests and $\chi^{2}$ or Fisher's exact tests were applied. Values were expressed as percentage and mean \pm SD. $P$ values $<0.05$ were considered statistically significant.
The protocol was approved by the ethics committee of Tabriz University of Medical Sciences, and informed consent was obtained from all the participants.

\section{Results}

Mean gestational age at recruitment was 12.2 weeks. There was no statistically significant difference in maternal BMI, maternal age, and nulliparity between the groups (Table 1). In the vitamin E group, $0.96 \%$ of women were complicated by preeclampsia in comparison with $1.8 \%$ in the control group (blood pressure $>140 / 90 \mathrm{mmHg}$ and 24-hour urine protein $>300 \mathrm{mg}$ ), with no statistically significant difference between the groups. The relative risk of preeclampsia was 0.54 .

Mean birth weight was $3187 \pm 545.09 \mathrm{~g}$ in the treated group and $3203.41 \pm 537.96 \mathrm{~g}$ in the control group $(P=0.8)$ (Table 2). Mean Apgar score at both 1-minute and 5-minute measurements was higher in the vitamin E group than the control group. The difference was not statistically different in any of the cases, but the $P$-value in 5-minute Apgar score was 0.09 vs 0.8 for 1-minute Apgar score. Term pregnancy was observed in $93 \%$ of vitamin E group compared with $90 \%$ of the control group.

\section{Discussion}

The possible positive effects of supplementary vitamin E during pregnancy have been studied previously by investigating changes in vitamin $\mathrm{E}$ levels in gestation or in normal vs problematic pregnancies. Oxidative stability of vitamin E levels was shown to increase in maternal blood during normal pregnancy. ${ }^{6}$ Also it has been shown that in some situations, such as smoking during pregnancy, the need for vitamin $\mathrm{E}$ may increase. ${ }^{7}$ In a study comparing abnormal and normal pregnancies, mean vitamin $\mathrm{E}$ levels rose from $12.9 \mu \mathrm{g} / \mathrm{mL}$ in early pregnancy to $22.5 \mu \mathrm{g} / \mathrm{mL}$ at term in normal pregnancies, but in pregnancies with fetal complications or maternal risks, levels were lower than in normal pregnancies at corresponding gestational age. ${ }^{8}$ In an earlier study by Tamura et al on 289 pregnant women in Birmingham, AL, no significant association was found between vitamin E serum concentrations and pregnancy outcomes. ${ }^{9}$

Table I Mean and standard deviation of the characteristics of mothers and their newborns in the studied groups

\begin{tabular}{llll}
\hline Group parameter & Vitamin E & Control & $P$-value \\
\hline Maternal age (years) & $25.29 \pm 5.14$ & $26.05 \pm 5.22$ & 0.6 \\
Nulliparous $(\%)$ & 60.8 & $5 \mathrm{I} .4$ & 0.4 \\
Maternal BMI $\left(\mathrm{kg} / \mathrm{m}^{2}\right)$ & $25.67 \pm 4.96$ & $27.8 \mathrm{I} \pm 5.1 \mathrm{I}$ & 0.9 \\
\hline
\end{tabular}


Table 2 Maternal and prenatal outcomes

\begin{tabular}{llllll}
\hline Group parameter & \multicolumn{4}{l}{ Vitamin E } & \multicolumn{2}{l}{ Control } & P-value \\
\hline Perinatal outcome & $\mathbf{N}$ & $\%$ & $\mathbf{N}$ & $\%$ & \\
Low birth weight $<2500 \mathrm{~g}$ & 8 & 7.6 & $\mathrm{II}$ & 6.5 & 0.6 \\
I-minute neonatal Apgar $<7$ & 4 & 3.8 & 10 & 5.9 & 0.26 \\
5-minute neonatal Apgar $<7$ & 0 & 0 & 4 & 2.4 & 0.26 \\
Maternal outcomes & & & & & \\
Cesarean section & 56 & 53.8 & 100 & 59.5 & 0.47 \\
Premature labor & 7 & 6.7 & 16 & 9.5 & 0.6 \\
\hline
\end{tabular}

An observational study showed that serum vitamin E level may be lower in pregnancies ending in a low-birth-weight delivery. ${ }^{8}$ Interestingly, however, two studies found that birth weight is significantly lower among those receiving supplementary vitamin $\mathrm{E}$ than in those not receiving it. ${ }^{10,11}$ We found that birth weight did not differ between those receiving vitamin $\mathrm{E}$ and those who did not. A meta-analysis of the few available clinical trials found that vitamin $\mathrm{E}$ supplementation did not affect birth weight. ${ }^{5}$ Although the available evidence does not seem to clearly support any association between vitamin $\mathrm{E}$ and birth weight, considering the paucity of information, possible heterogeneity in the available studies, and statistical power limitations, further studies are needed to support stronger conclusions in this regard.

Preeclampsia is another outcome of interest. A small study found that the concentrations of vitamin $\mathrm{E}$ was significantly lower in a preeclampsia group than in a normotensive group at 28 weeks' gestation. ${ }^{12}$ Another observational study found total antioxidant and vitamin C concentrations to be significantly lower in preeclamptic patients than in pregnant controls. ${ }^{13}$ No significant difference in vitamin $\mathrm{E}$ concentration was found among four groups (normotensives, mild preeclamptics, severe preeclamptics, and healthy nonpregnant women), but the groups were not large enough to support a strong conclusion. Nevertheless, several similar clinical trials have also been conducted leading to some controversy in this regard, but most were not in favor of the effect in preventing preeclampsia. Our findings do not support the hypothesis that vitamin $\mathrm{E}$ (400 IU) supplements, given prophylactically in pregnancy, reduce the rate of preeclampsia: the rate of preeclampsia in the treated group was similar to that in the control group. However, our study lacks adequate statistical power to make a strong conclusion and any interpretation based on these findings should be made cautiously. Several other trials have examined the effectiveness of vitamins $\mathrm{C}$ and $\mathrm{E}$ in preventing preeclampsia, using the same doses of vitamin $\mathrm{E}$ as in our trial. Several studies have shown no significant benefits of administering antioxidants to pregnant women. ${ }^{5,11,14}$ In contrast, Chappell et al found that treatment from early pregnancy (16 to 22 weeks) with a combination of $1000 \mathrm{mg}$ vitamin C and $400 \mathrm{IU}$ vitamin E daily in a highrisk population can significantly $(17 \%)$ reduce preeclampsia rate. ${ }^{15}$ However, the need to investigate the optimum dosing and timing of supplementary antioxidant administration has been discussed, since inappropriate antioxidant treatment in pregnant women may reduce placental cell proliferation and even lead to cell death. ${ }^{16}$ In a multicenter randomized controlled trial, in which 2647 women were assigned to daily treatment ( $1 \mathrm{~g}$ vitamin $\mathrm{C}$ and $400 \mathrm{IU}$ vitamin $\mathrm{E}$ ) or placebo, vitamin $\mathrm{C}$ and $\mathrm{E}$ supplementation did not reduce the rate of preeclampsia or gestational hypertension, but increased the risk of fetal loss or perinatal death and preterm prelabor rupture of membranes. ${ }^{17}$

In all these studies, the combined effect of vitamin $\mathrm{C}$ and $\mathrm{E}$ has been assessed, while the present study evaluated the effect of vitamin E supplementation in isolation on preeclampsia. Vitamin E supplementation is not reported to be generally contraindicated in pregnancy. Although vitamin $\mathrm{E}$ has not been reported to have major detrimental effects on preterm neonates or pregnant women, lower birth weight in women receiving vitamin $\mathrm{E}$ has been reported. ${ }^{10,15}$ However, vitamin $\mathrm{E}$ has been shown to have positive effects on both the mother and the neonate in some conditions. ${ }^{18,19}$ Numbers of adverse perinatal outcomes were small in this study, but were similar in both groups.

Consistent with most other published studies, our results also do not support the efficacy of supplementary vitamin E to improve pregnancy outcomes or prevent preeclampsia.

Although our descriptive findings on the effect of vitamin $\mathrm{E}$ in preventing preeclampsia were in line with previous studies, lower statistical power should be considered as an explanation in the interpretation of the results. Biochemical assessments were not performed in this study, but randomization techniques likely assure the randomness of confounding in this study.

\section{Acknowledgments}

This investigation was supported by the research deputy of Tabriz University of Medical Sciences. We also thank Mrs Abbasnezhad, Mrs Delghoshamehr, and Mrs Pourshiri for their assistance in performing this study.

\section{Disclosure}

The authors declare no conflicts of interest in this work. 


\section{References}

1. Carty DM, Delles C, Dominiczak AF. Preeclampsia and future maternal health. J Hypertens. 2010;28(7):1349-1355.

2. Evans HM, Bishop KS. On the existence of a hitherto unrecognized dietary factor essential for reproduction. Science. 1922;56(1458):650-651.

3. Brigelius-Flohe R. Vitamin E: the shrew waiting to be tamed. Free Radic Biol Med. 2009;46(5):543-554.

4. Siddiqui IA, Jaleel A, Tamimi W, Al Kadri HM. Role of oxidative stress in the pathogenesis of preeclampsia. Arch Gynecol Obstet. 2010;282(5): 469-474.

5. Rumbold A, Crowther CA. Vitamin E supplementation in pregnancy. Cochrane Database Syst Rev. 2005;(2):CD004069.

6. De V Sr, Dhont M, Christophe AB. Oxidative stability of low density lipoproteins and vitamin $\mathrm{E}$ levels increase in maternal blood during normal pregnancy. Lipids. 2001;36(4):361-366.

7. Chelchowska M, Laskowska-Klita T, Leibschang J. The effect of tobacco smoking during pregnancy on concentration of vitamin $\mathrm{E}$ in blood of mothers and their newborns in umbilical cord blood. Ginekol Pol. 2006;77(4):263-268.

8. von MU, Huch R, Huch A. Maternal and cord serum vitamin E levels in normal and abnormal pregnancy. Int J Vitam Nutr Res. 1994;64(1): $26-32$.

9. Tamura T, Goldenberg RL, Johnston KE, Cliver SP, Hoffman HJ. Serum concentrations of zinc, folate, vitamins A and E, and proteins, and their relationships to pregnancy outcome. Acta Obstet Gynecol Scand Suppl. 1997;165:63-70.

10. Boskovic R, Gargaun L, Oren D, Djulus J, Koren G. Pregnancy outcome following high doses of Vitamin E supplementation. Reprod Toxicol. 2005;20(1):85-88.
11. Poston L, Briley AL, Seed PT, Kelly FJ, Shennan AH. Vitamin C and vitamin $\mathrm{E}$ in pregnant women at risk for pre-eclampsia (VIP trial): randomised placebo-controlled trial. Lancet. 2006;367(9517): 1145-1154.

12. Jendryczko A, Drozdz M. Plasma retinol, beta-carotene and vitamin E levels in relation to the future risk of pre-eclampsia. Zentralbl Gynakol. 1989;111(16):1121-1123.

13. Bowen RS, Mars M, Chuturgoon AA, Dutton MF, Moodley J. The response of the dietary anti-oxidants vitamin $\mathrm{E}$ and vitamin $\mathrm{C}$ to oxidative stress in pre-eclampsia. J Obstet Gynaecol. 1998;18(1):9-13.

14. Roberts JM, Myatt L, Spong CY, et al. Vitamins C and E to prevent complications of pregnancy-associated hypertension. $N$ Engl J Med. 2010;362(14):1282-1291.

15. Chappell LC, Seed PT, Briley AL, et al. Effect of antioxidants on the occurrence of pre-eclampsia in women at increased risk: a randomised trial. Lancet. 1999;354(9181):810-816.

16. Beazley D, Ahokas R, Livingston J, Griggs M, Sibai BM. Vitamin C and $\mathrm{E}$ supplementation in women at high risk for preeclampsia: a doubleblind, placebo-controlled trial. Am J Obstet Gynecol. 2005;192(2): 520-521.

17. Xu H, Perez-Cuevas R, Xiong X, et al. An international trial of antioxidants in the prevention of preeclampsia (INTAPP). Am J Obstet Gynecol. 2010;202(3):239.

18. Devereux G, Turner SW, Craig LC, et al. Low maternal vitamin E intake during pregnancy is associated with asthma in 5-year-old children. $\mathrm{Am}$ J Respir Crit Care Med. 2006;174(5):499-507.

19. Fruscella L, Ciaglia EM, Danti M, Fiumara D. Vitamin E in the treatment of pregnancy complicated by uterine myoma. Minerva Ginecol. 1997;49(4):175-179.
International Journal of General Medicine

\section{Publish your work in this journal}

The International Journal of General Medicine is an international, peer-reviewed open-access journal that focuses on general and internal medicine, pathogenesis, epidemiology, diagnosis, monitoring and treatment protocols. The journal is characterized by the rapid reporting of reviews, original research and clinical studies across all disease areas.

\section{Dovepress}

A key focus is the elucidation of disease processes and management protocols resulting in improved outcomes for the patient.The manuscript management system is completely online and includes a very quick and fair peer-review system. Visit http://www.dovepress.com/ testimonials.php to read real quotes from published authors. 